\title{
Modern Solid state Laser Materials
}

\author{
Wiltiam F. Krupke
}

\section{DISCLAIMER}

This report was prepared as an accounl of wouk sponsored by an agescy of the United States Goverment. Neitber the United Stotes úbvernment nor any agency thereor, abs aby of their emplayees, makes any warranty, express or implied, or assumes any lesal liability or tesponsibility for the accuracy, compleleness, or uselulness of any inforthation, apparatus, product, or prosess disclased, or represents that its use wauld not infringe privately owned rights. Refer-

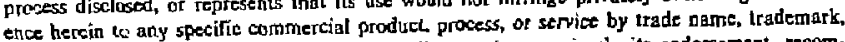

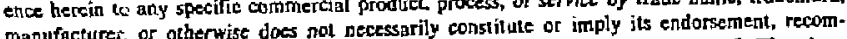
mandation. of favoring by the Uniled States Governinemt or any agency thereof. The views and opinions of authors expressed herein do not nexessarily state or reflect thase of the United States Government ar any agency thereor.

Jine 20,1984

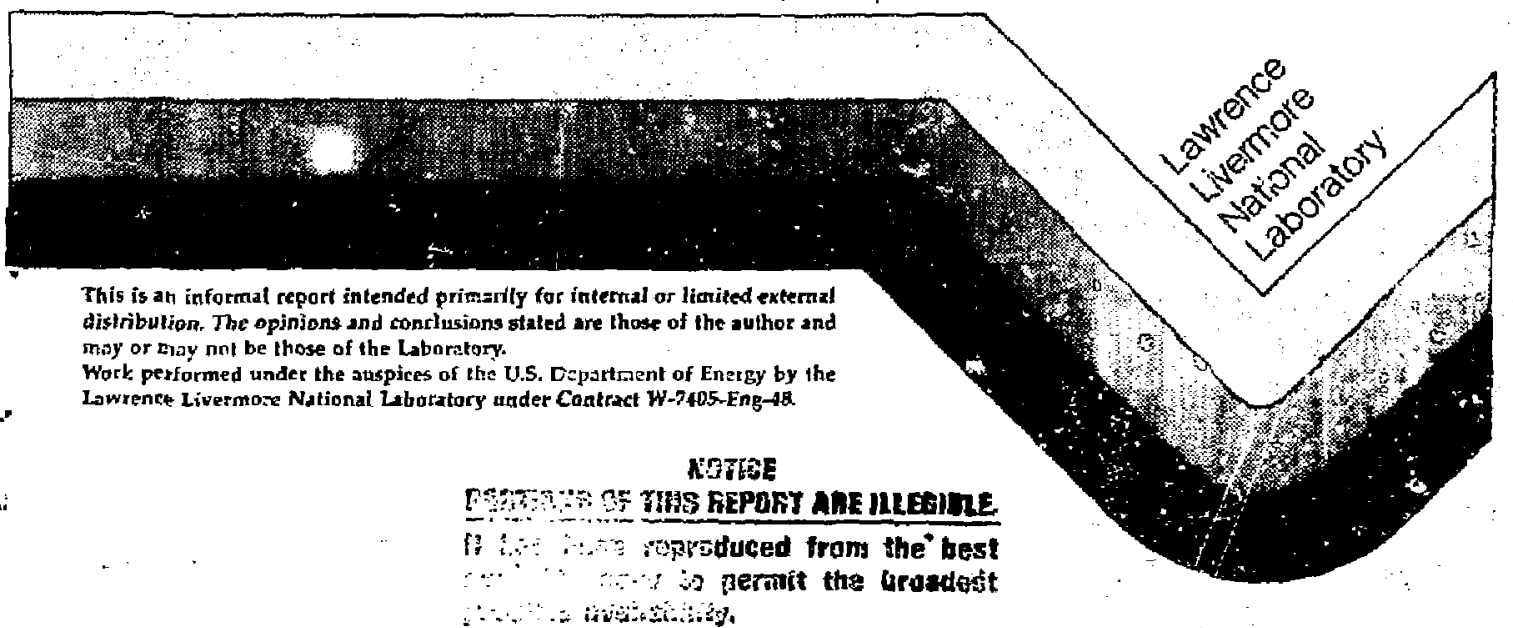


Modern Solid State Laser Materials

by

UCID- 20110

William F. Krupke

DE85 003665

This document contains visual aids used in an invited talk entitled "Madern Solid State Laser Materials," presented at the Conference on Lasers and Electro-Optics (CLEO) held in Anaheim, California, on June 20, 1984. Collaborators in the work presented include Howard Guggenheim of AT\&T Bell Laboratories, Murray Hill, New Jersey; Frank Bruni of Materials Progress Corporation, Santa Rosa, Califoriaia and colleagues at Lawrence Livermore National Laboratory (LLNL): John Caird, Herbert Newkirk, Michael Shinn, Stanley Stokowski, and Ray Wilder. Interest ai LLNL in solid state lasers focuses on evaluating the potential of solid state laser media for high average power applications, including inertial fusion powar production. To De successful, solid state laser materials will have to attain higher performance levels of energy-storage density, efficiency, and aperture scaling than heretofore. This talik identifies the relevant bulk material parameters characterizing average power capacity and uses chromium and neodymium co-doped gadol inium scandium gallium garnet (Nd:Cr:GSGG) as an example of a Jaser material with improved laser properties relative to Nd:YAG (plausible large-scale growth, more efficient spectral coupling to xenon flashlamp radiation, reduced stimulated emission cross stion, adequate thermal shock and optical damage threshoid parameters, etc.). Recently measured spectroscupic, kinetic, and thermo-mechanical properties of Nd:Cr:GSGG are given.

Tunable lasers based on phonon-terminated transitions of transitionmetal doped solids is of current interest with respect to a variety of applications. We have recently observed stimulated emission in the phonon-terminated ${ }^{4} \mathrm{~T}_{2} \rightarrow{ }^{4} \mathrm{~A}_{2}$ band of $\mathrm{Cr}^{3+}$ in two fluoride crystalline host materials doped with trivalent chromium: the fiuoride garnet $\mathrm{Cr}: \mathrm{Li}_{3} \mathrm{Na}_{3} \mathrm{Ga}_{2} \mathrm{~F}_{12}$ and the fluoride perovskite $\mathrm{G}: \mathrm{KZnF}{ }_{3}$.

To be efficlent as a short pulse laser (to enable subsequent nonlinear wavelength shifting operations), one desires a stimulated emission cross

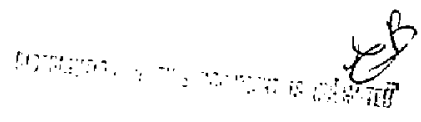


section of a few times $10^{-20} \mathrm{~cm}^{2}$. This value will permit the stored eriergy to be efficiently cxtracted at a beam fluence below the optical damage fluence. At the same time, one desires an energy storage lifetime of at least 100 usec so that flashlamp pumping can be carried out with reasonably high lamp shot life. These requirements are more readily realizable in fluoride based materials than in oxides because of the significantly lower index of refraction of the former compared to the latter. In this respect, the system $\mathrm{Cr}_{\mathrm{K} Z \mathrm{KnF}}$ offers an improved set of laser spectroscopic parameters for efficient tunable laser action near $800 \mathrm{~nm}$. While this material (indeed most fluorides) possesses less rugged thermo-mechanical properties compared to many oxide crystais, it may prove to be sufficiently robust for a number of useful applications. Measurement of relevant physical properties are currently in progress. Forma: reports describing the measurements and data presented in this talk are in preparation currently. 
Modern solid state laser materials - topics

Cr:Nd:Gadolinium oxide garnets

- Spectroscopy

- Laser parameters

- Thermo-mechanical properties

- Growth

Cr-tunable laser materials

- Fluoride/oxide garnets

- Fluoride perovskites/elpasolites

- Laser parameters 
Cr-Nd Co-doped materials

D. Pruss, et al.

E.V. Zharikov, et al.

E. $\forall$. Zharikov, et al.
Appl. Phys. B28, 355 (1982).

Sov. J. Quantum Electron. 12, 338 (1982).

Sov. J. Quantum Electron. 13, 82 (1983).

Cr-doped tunable materials

B. Struve, et al.

B. Struve, et al.

$E_{\text {.V. Z }}$ Zharikov。 et al.

U. Brauch, et @.
Appi. Phys. B28, 235 (1982). Appl. Phys. B30, 117 (1983). Sov. J. Quantum Electron. 13, 1274 (1983). Optics Communications 49, 61 (1984). 


\section{Modern crystailine lasers - collaborators}

AT\&T Bell l-aboratories

Howard Guggenheim

Materials Progress, Inc.

Frank Bruni

\begin{tabular}{l} 
LLNL \\
\hline John Caird \\
Herb Newkirk \\
Mike Shinn \\
Stan Stokowski \\
Ray Wilder
\end{tabular}

02-50-0684-1945 


\section{High average power laser amplifier geometries}
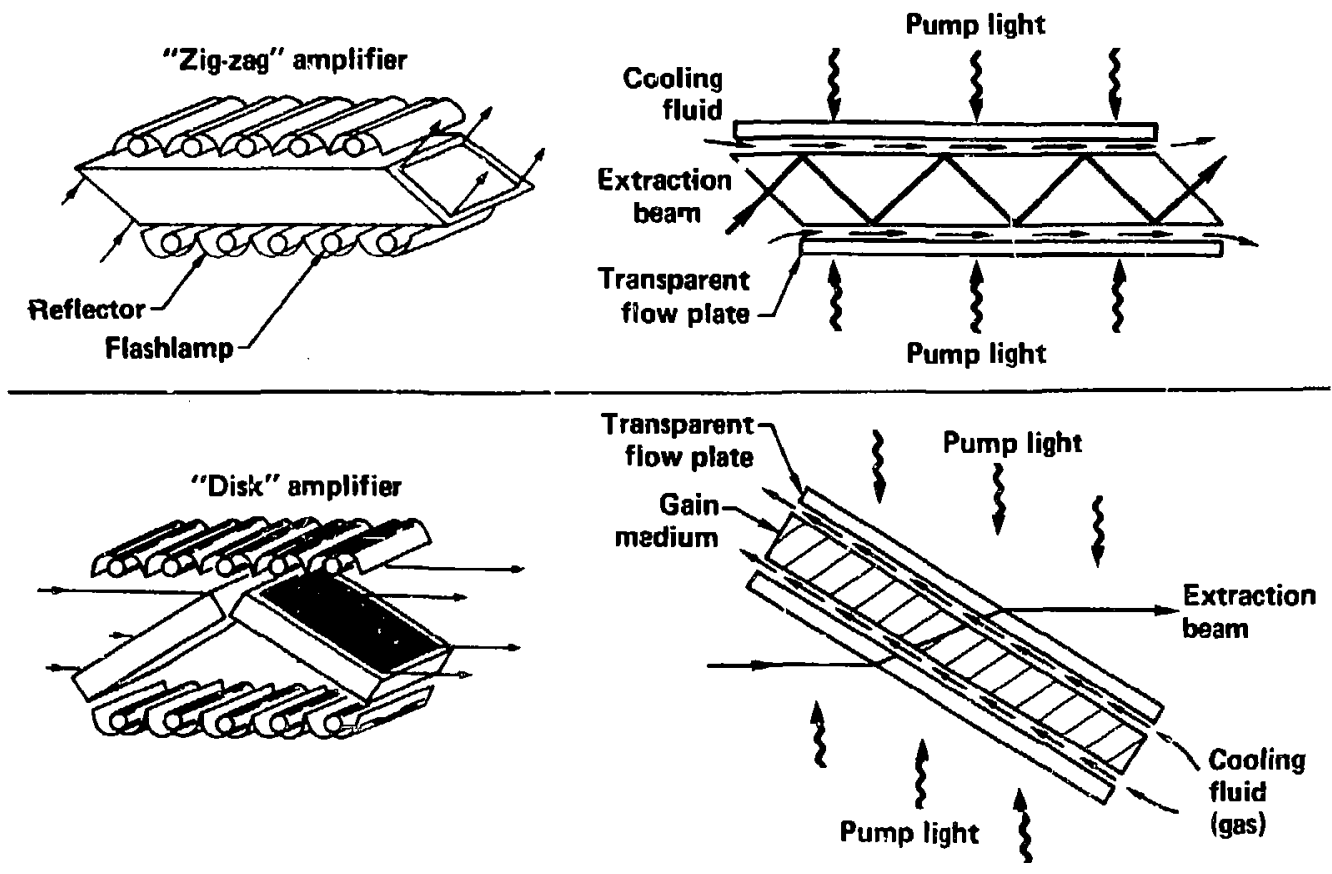


\section{Thermal power capacity of a}

\section{uniformly-heated flow-cooled plate}

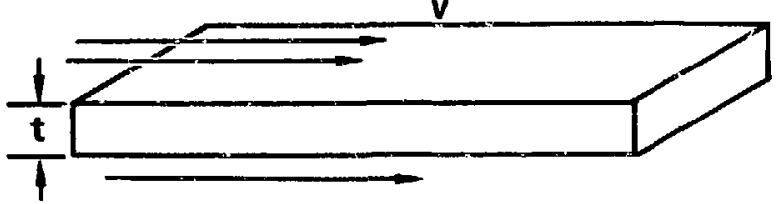

$\frac{\text { Therimal power }}{\text { Unit volume }}=\mathrm{F}_{\mathrm{V}}=\frac{12 \mathrm{R}_{\mathrm{T}} \mathrm{b}}{\mathrm{t}^{2}} \approx(0.5-2)\left\{\frac{\text { Optical power }}{\text { Unit volume }}\right\} ; \mathrm{R}_{\mathrm{T}}=\frac{(1-\nu) \kappa \mathrm{S}_{\mathrm{T}}}{\alpha \mathrm{E}}$

$\mathbf{R}_{\mathbf{T}}=$ Thermal shock parameter Three high power strategies:

$\nu \quad=$ Poisson's ratio

- Materials with high $\mathbf{R}_{\mathrm{T}}$ :

$\kappa=$ Thermal conductivity

- High $\kappa \Rightarrow$ crystals

$\mathbf{S}_{\boldsymbol{T}} \quad=$ Tensile yield strength

$\alpha \quad=$ Thermal expansion coefficient

- Low $\alpha \Rightarrow\left\{\begin{array}{l}\text { Low expansion glasses } \\ \text { Glass ceramics }\end{array}\right.$

E $\quad$ Young's modulus

- Small $\mathbf{t} \Rightarrow$ Composite plate

b $\quad=\frac{\text { Thermally induced stress }}{\text { Tensile yield stress }}$ structures 


\section{$3 d^{3}-$ Crystal field interactions}
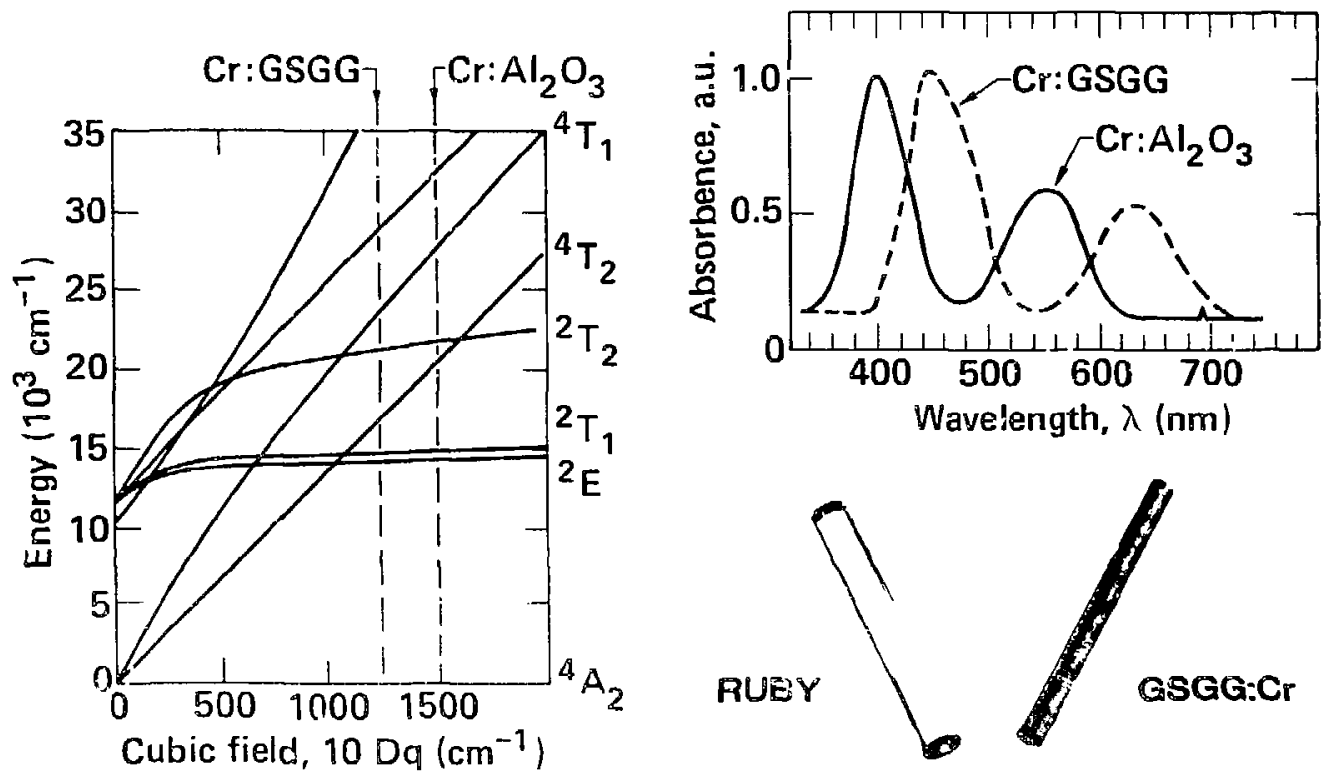

RUEY
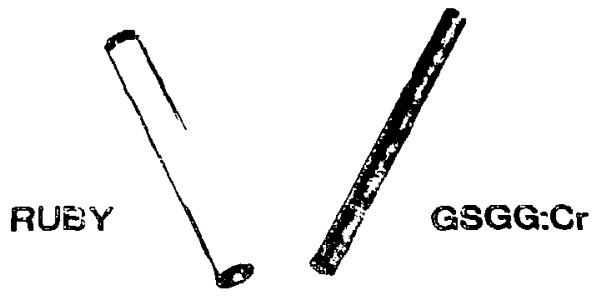

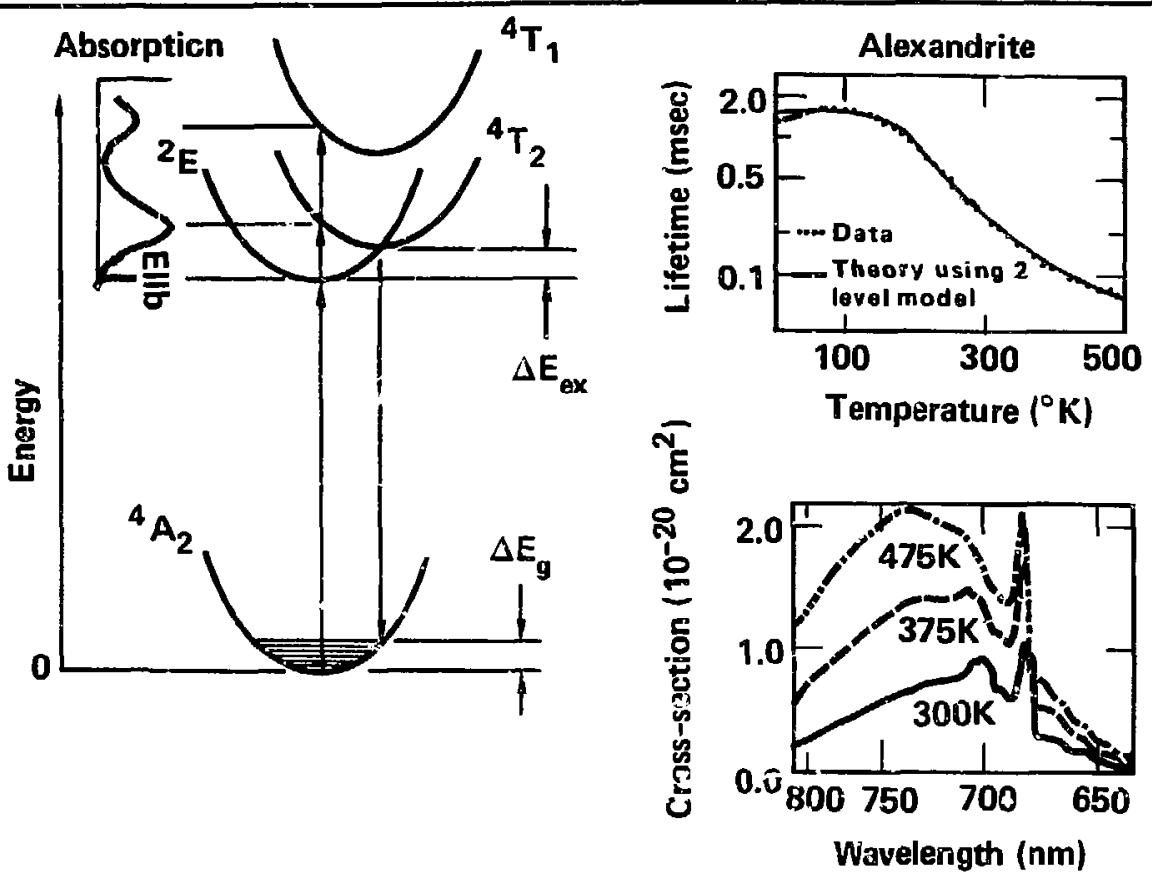


\section{Systematics of $\mathrm{Cr}$ :Garnet ${ }^{4} \mathrm{~T}_{2}$ fluorescence}

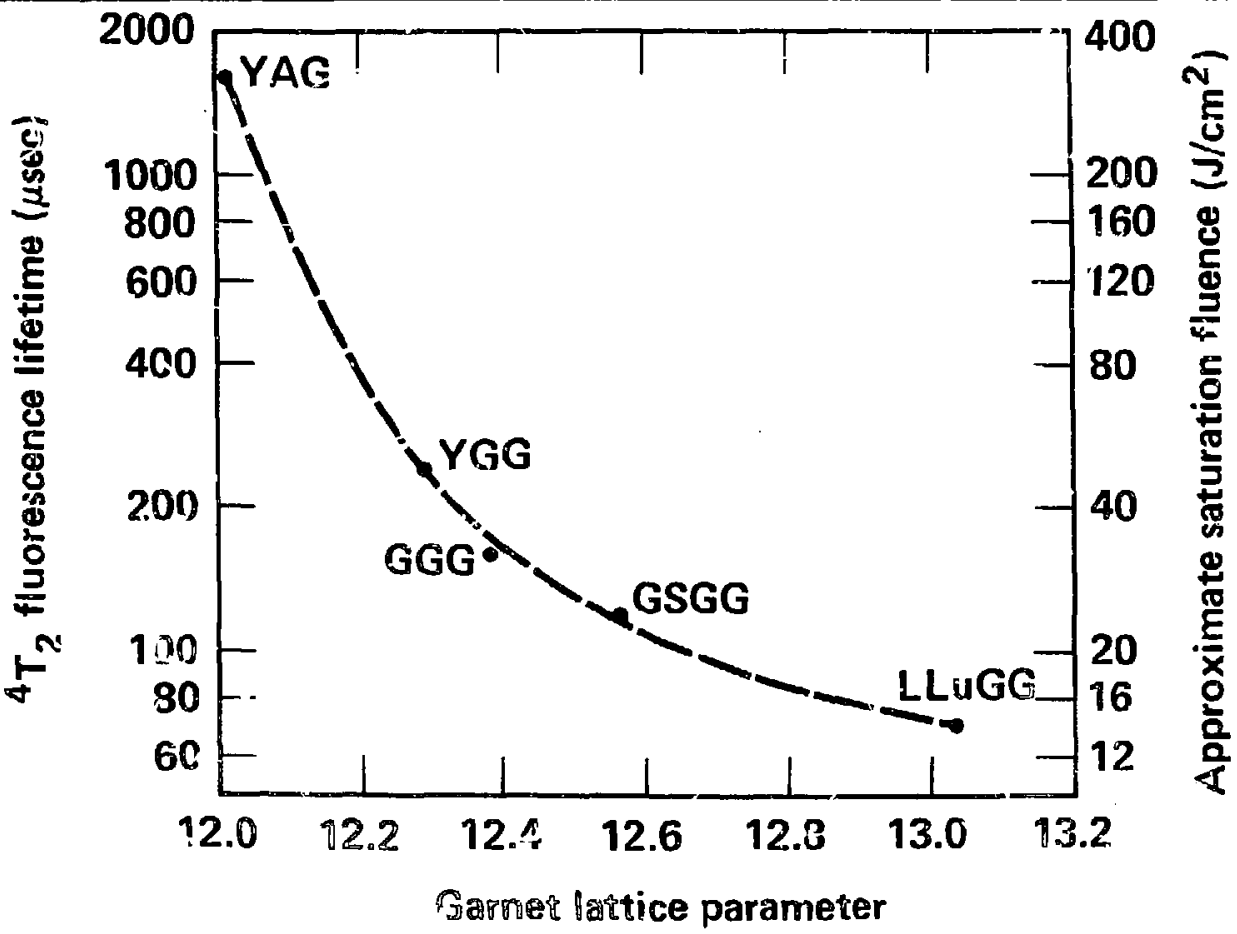




\section{$\mathrm{Cr} \rightarrow \mathrm{Nd}$ sensitization laser}

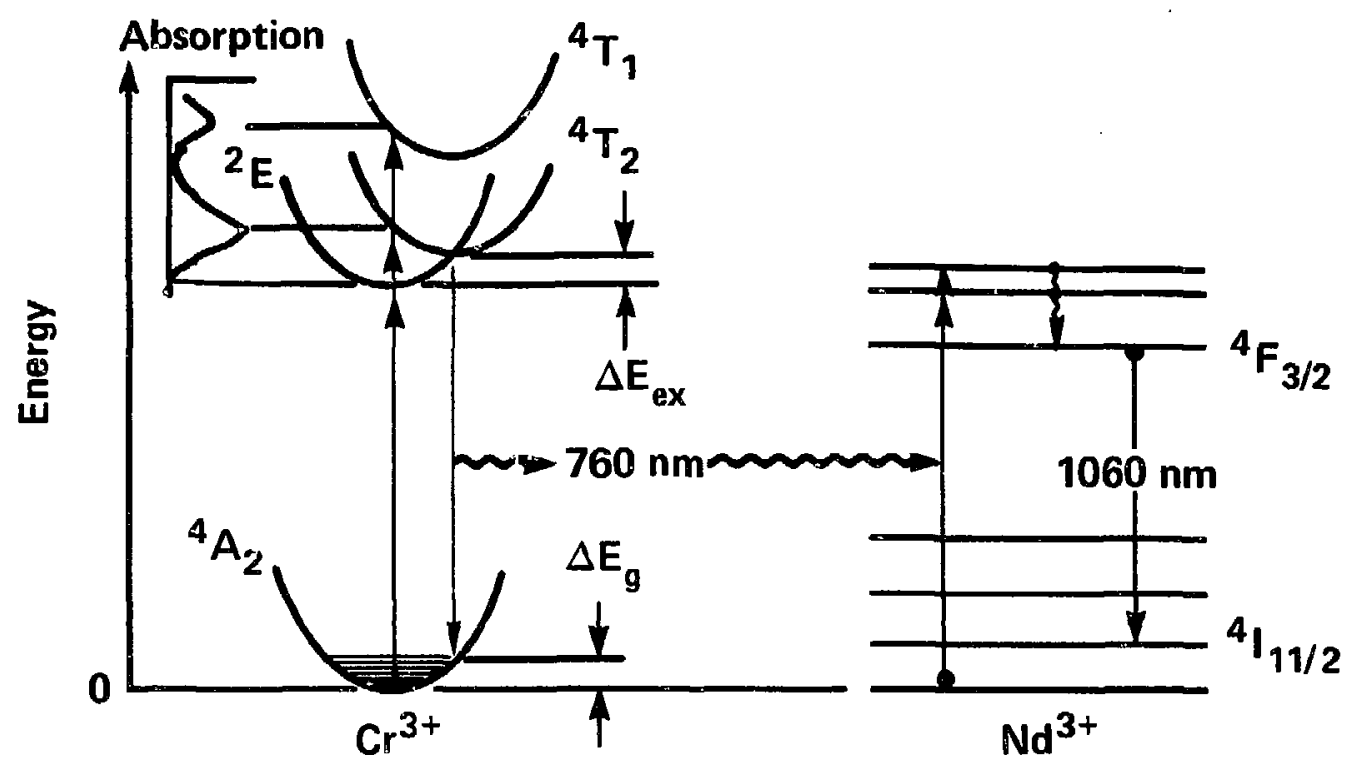




\section{Absorbance - Nd:Cr:GSGG}

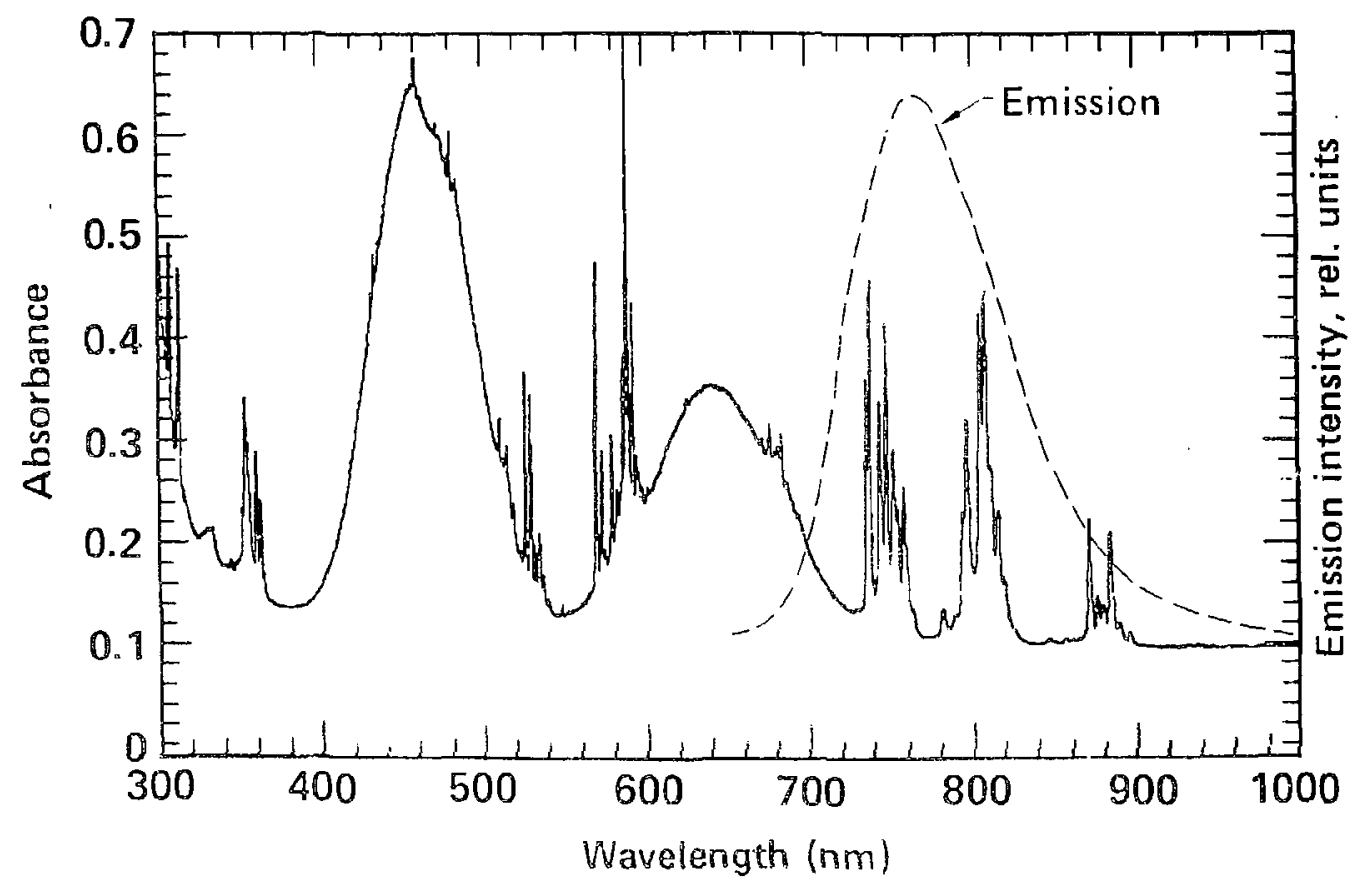

$02 \cdot 50 \cdot 06841969$ 


\section{$\mathrm{Cr} \rightarrow \mathrm{Nd}$ transfer efficiency in GSGG}

Data: Zharikov, etal. Sov. J.Q.E. 13, 82 (1983); 12, 338 (1982).

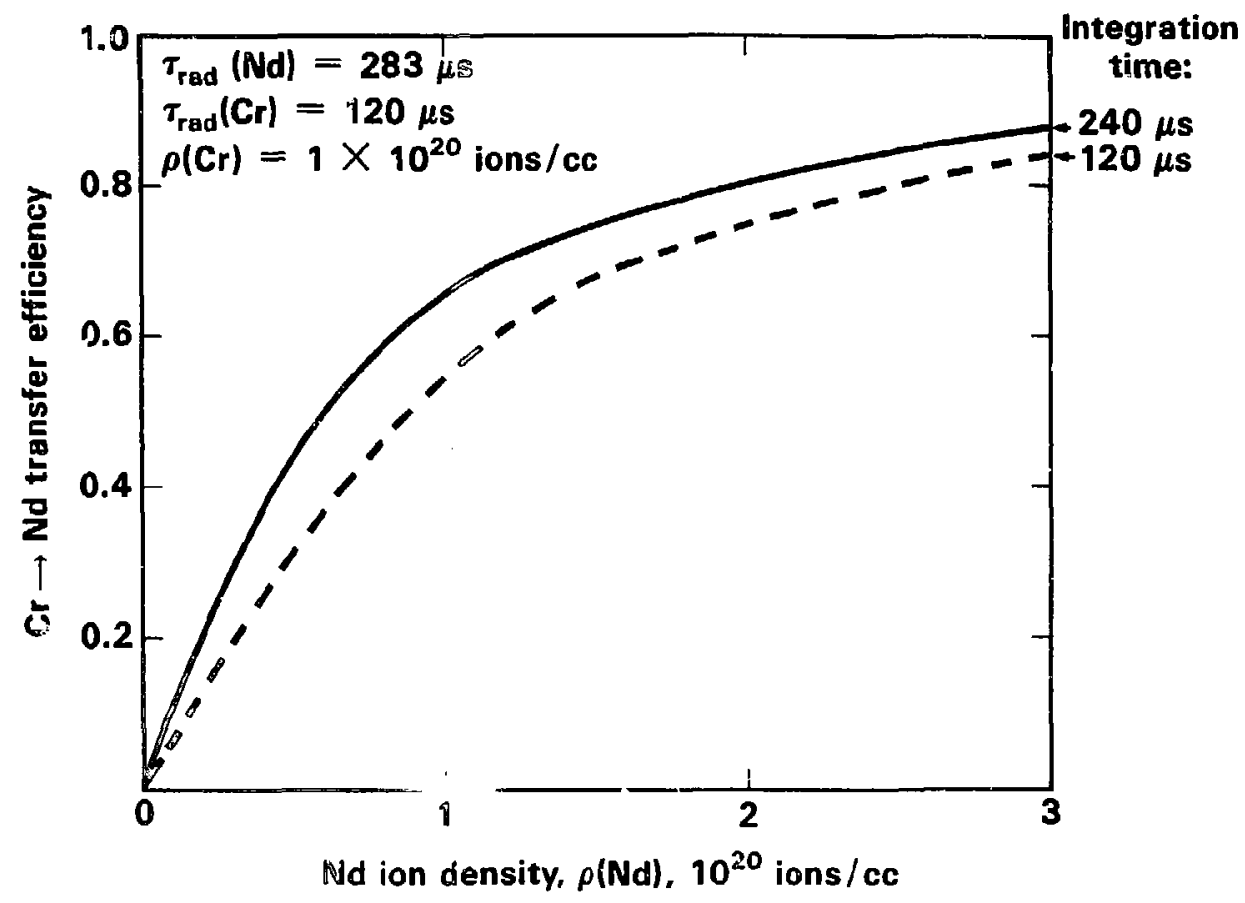




\section{${ }^{4} F_{3 / 2}$ excitation energy efficiency (xenon flashlamp)}

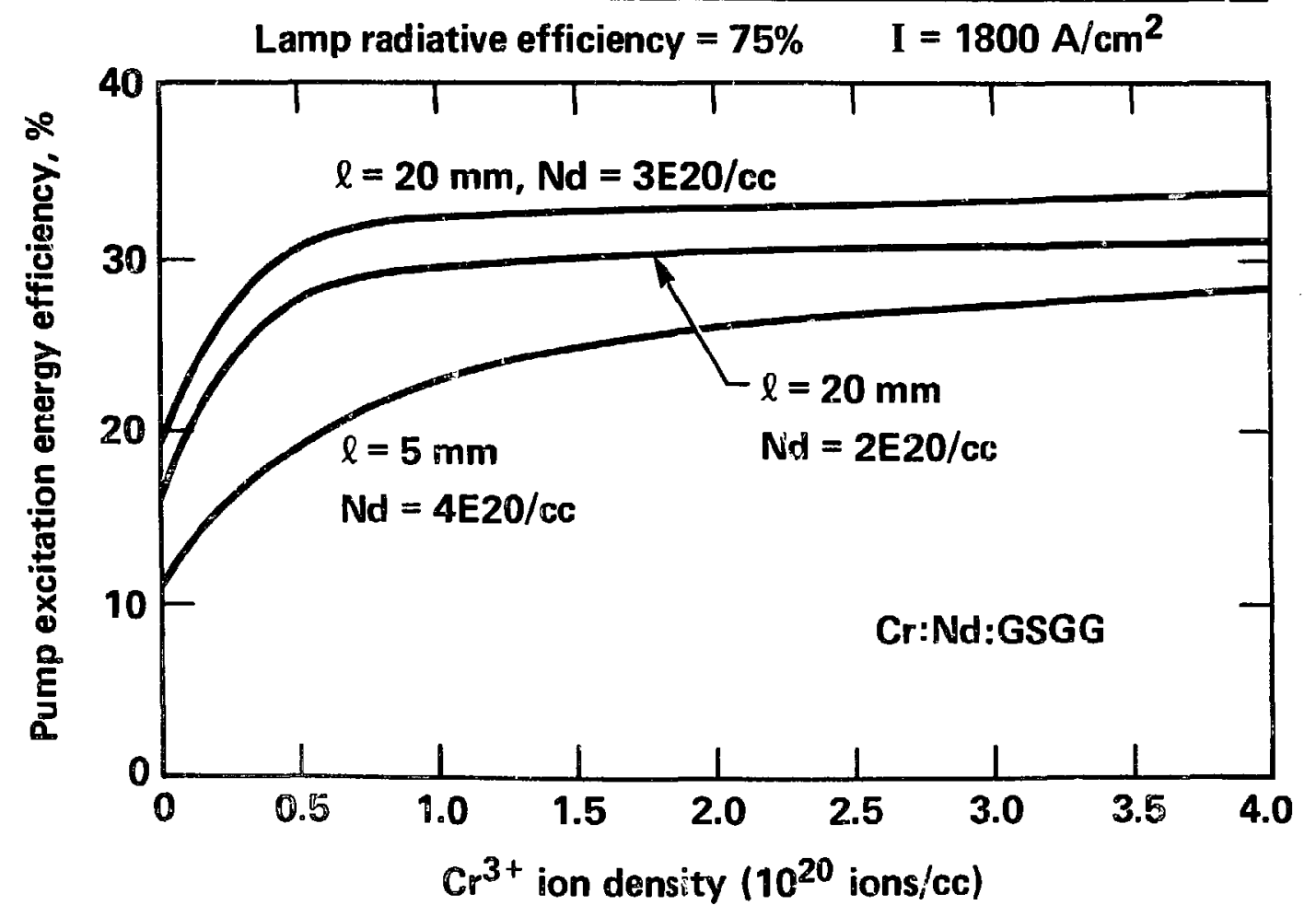




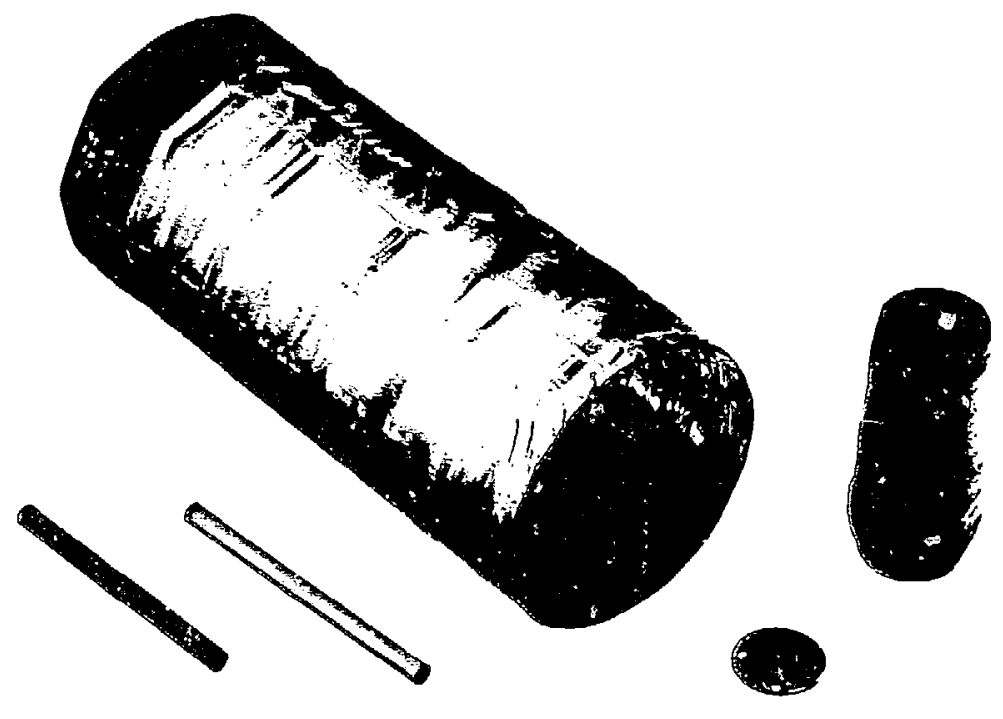

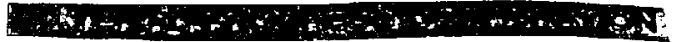

* Growth: F. Bruni (Material Progress Corp.)

0? $50 \cdot 0684 \cdot 1989$ 


\section{Oxide garnet laser materials*}

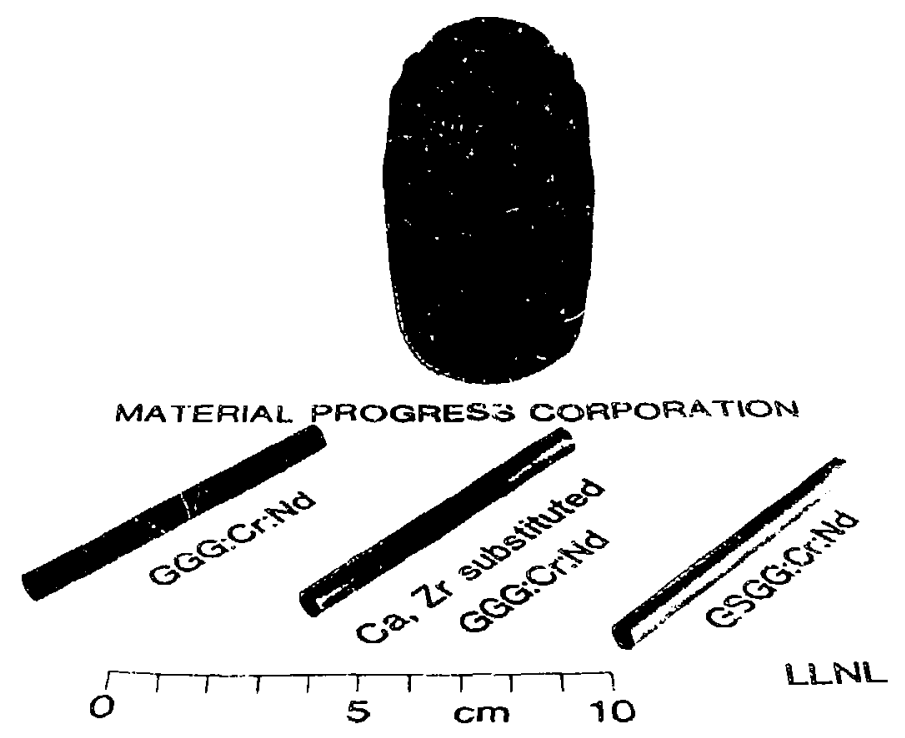

* Growth: F. Bruni (Material Progress Corp.)

$02500684 \cdot 1985$ 
${ }^{4} F_{3 / 2} \rightarrow{ }^{4} I_{11 / 2}$ fluorescence - oxide garnets $\left(300^{\circ} \mathrm{K}\right)$

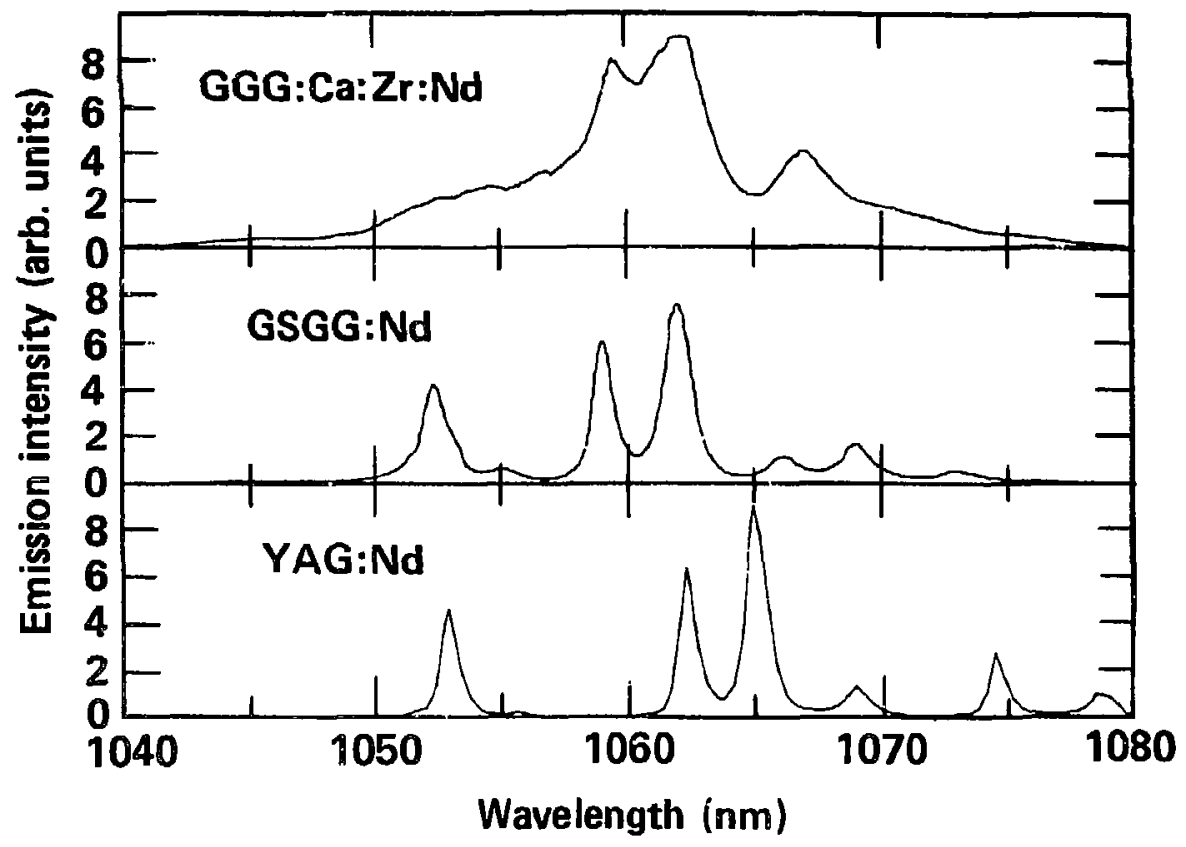


Nd:GSGG - fluorescence-concentration quenching

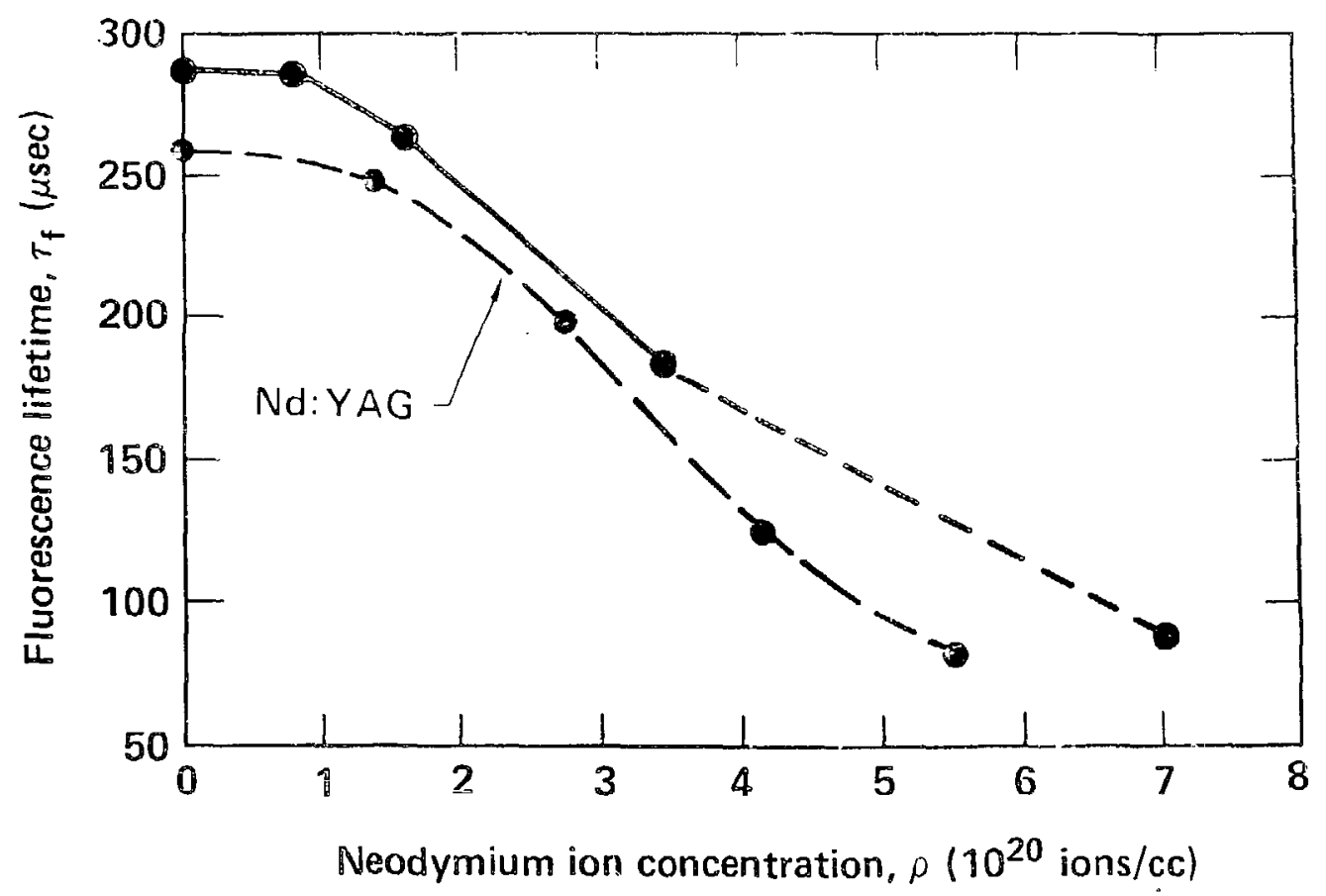




\section{Spectroscopic laser parameters (I)}

Property

Judd-Ofelt param. $\Omega_{2}, \Omega_{4}, \Omega_{6}\left(10^{-20} \mathrm{~cm}^{2}\right)$

${ }^{4} F_{3 / 2}$ - J.O. caic. radiative lifetime

Zero concentration lifetime

Half-lifetime quenching conc.

${ }^{4} F_{3 / 2}$ Stark splitting

$R_{2} \rightarrow Y_{3}$ peak wavelength

$R_{2} \rightarrow Y_{3}$ peak cross-section, $\sigma_{L}$

Effective small-signal gain cross section ${ }^{\dagger}, \sigma_{\text {eff }}$

Homogeneous saturation fluence*

* Does not account for orientational hole-burning.

$t_{\alpha}=\sigma_{\text {eff }} \Delta \mathbf{N}\left({ }^{4} \mathbf{F}_{3 / 2}\right)$
Nd:GSGG

Nd:YAG

$0.35,2.35,3.23$

$0.2,2.7,5.0$

$283 \mu \mathrm{sec}$

$259 \mu$ sec

$286 \mu$ sec

$\sim 260 \mu \mathrm{sec}$

$\sim 5 \times 10^{20}$ ions/cc $\sim 4 \times 10^{20}$ inns/cc

$60 \mathrm{~cm}^{-1}$

$84 \mathrm{~cm}^{-1}$

$1061 \mathrm{~nm}$

$1064 \mathrm{~nm}$

3. $2 \times 10^{-19} \mathrm{~cm}^{2}$

$7 \times 10^{-19} \mathrm{~cm}^{2}$

$1.4 \times 10^{-19} \mathrm{~cm}^{2}$

$2.8 \times 10^{-19} \mathrm{~cm}^{2}$

$1.0 \mathrm{~J} / \mathrm{cm}^{2}$

$0.5_{0} \mathrm{~J} / \mathrm{cm}^{2}$ 
Property

${ }^{4} \mathrm{~A}_{2} \rightarrow{ }^{4} \mathrm{~T}_{2}$ peak wavelength

${ }^{4} \mathrm{~A}_{2} \rightarrow{ }^{4} \mathrm{~T}_{2}$ peak cross-section, $\sigma_{A}$

${ }^{4} \mathrm{~T}_{2} \rightarrow{ }^{4} \mathrm{~A}_{2}$ peak cross-section, $\sigma_{E}$

${ }^{4} T_{2}$ fluorescence lifetime

Half-lifetime quenching conc.

\section{Cr:GSGG}

$640 \mathrm{~nm}$

$3.3 \times 10^{-20} \mathrm{~cm}^{2}$

$0.8 \times 10^{-20} \mathrm{~cm}^{2}$

$120 \mu \mathrm{sec}$

$>3 \times 10^{20}$ ions/cc 


\section{GSGG material properties (preliminary)}

Property

Thermal conductivity, $k$

Thermal expansion, $\alpha$

Young's modulus, $\mathrm{E}$

Breaking strength ${ }^{\dagger}$ (4-pt bend), $S_{T}$

Poisson's ratio, $v$

Thermal shock*, $\mathbf{R}_{\mathbf{T}}$

Index of refraction, $1060 \mathrm{~nm}$

Censity

Nd-segregation coeff.

Cr-segregation coeff.

TO ns damage threshold

${ }^{n} \mathrm{R}_{\mathrm{T}}=(1-\nu) \kappa \mathrm{S}_{\mathrm{T}} / \alpha \mathrm{E}$

tDepends on fabrication
Cr:Nd:GSGG

7-9 $\mathrm{w} / \mathrm{m}^{\circ} \mathrm{C}$ (conc. dep.)

$$
7.8 \times 10^{-6} /{ }^{\circ} \mathrm{C}
$$$$
-21,000 \mathrm{~kg} / \mathrm{mm}^{2}
$$

$15-20 \mathrm{~kg} / \mathrm{mm}^{2}$

$0.11-0.18$

$\sim 700 \mathrm{~W} / \mathrm{m}$

1.925

6.46

$0.65 \pm 0.04$

$1.0^{-}$

$\left\{\begin{array}{l}16 \mathrm{~J} / \mathrm{cm}^{2}, \text { surface } \\ >28 \mathrm{~J} / \mathrm{cm}^{2}, \text { bulk }\end{array}\right.$
Nd:YAG

$\sim 13 \mathrm{~W} / \mathrm{m}^{\circ} \mathrm{C}$

$7.8 \times 10^{-6} /{ }^{\circ} \mathrm{C}$

$\sim 31,700 \mathrm{~kg} / \mathrm{mm}^{2}$

$\sim 20 \mathrm{~kg} / \mathrm{mm}^{2}$

Not reported

$\sim 800 \mathrm{~W} / \mathrm{m}$

1.815

4.55

0.18 


\section{Laser performance* $-\mathrm{Cr}: \mathrm{Nd}: \mathrm{GSGG}$}

Long-pulse $(R=0.66) \quad \phi=5 \mathrm{~mm} \quad I=50 \mathrm{~mm}$

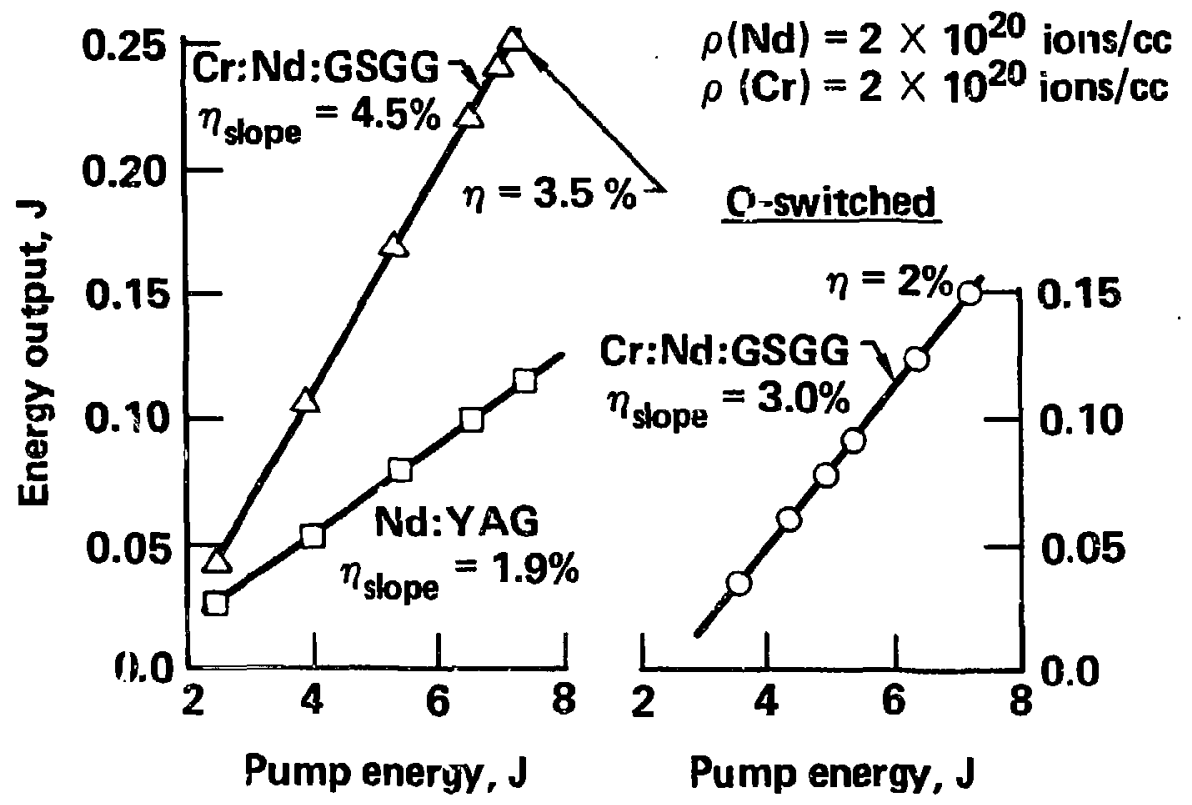

*E.V. Zharikov ${ }^{\circ}$ et al., Sov. J. Quantum Electron., 13, 1306 (1983). 


\section{$1000 \mathrm{~nm}$ loss in selected Cr:Nd:GSGG crystals}

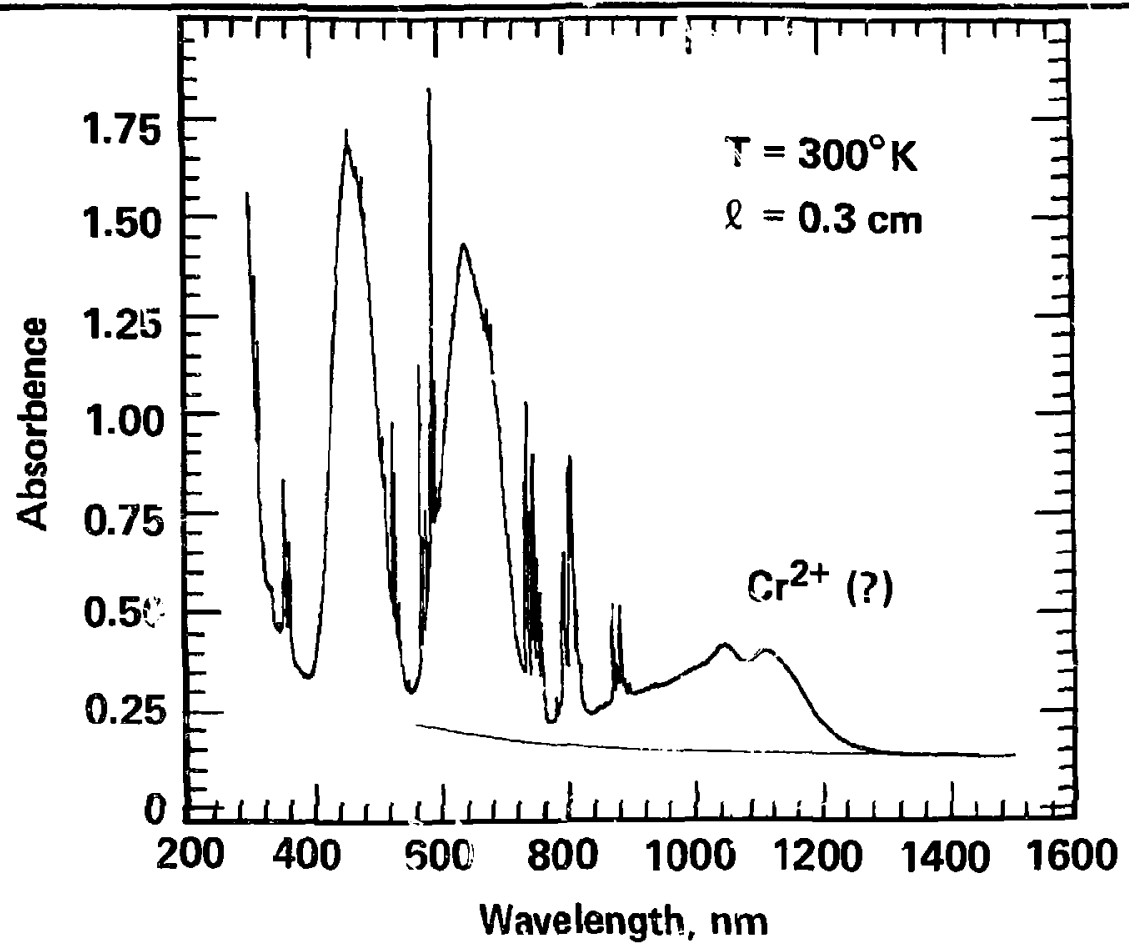




\section{Tunable chromium-doped laser materials}

\begin{tabular}{|c|c|c|c|}
\hline Crystal Type & Co: iposition & $\begin{array}{c}\text { Peak } \\
\text { Fluor.- } \lambda \\
\text { (nm) }\end{array}$ & Pimp Sources \\
\hline Oxide garnet & $\mathrm{G}: \mathrm{d}_{3} \mathrm{Ga}_{2} \mathrm{Ga}_{3} \mathrm{O}_{12}$ & 760 & $\mathbf{K r}$ \\
\hline Oxide garnet & $\mathrm{Gd}_{3} \mathrm{Sc}_{2} \mathrm{Ga}_{3} \mathrm{O}_{12}$ & 770 & Kr; flashlamp \\
\hline Oxide garnet & $\mathrm{Y}_{3} \mathrm{Ga}_{2} \mathrm{Ga}_{3} \mathrm{O}_{12}$ & 740 & $\mathbf{K r}$ \\
\hline Oxide garnet & $\mathrm{Y}_{3} \mathrm{Sc}_{2} \mathrm{Ga}_{3} \mathrm{O}_{12}$ & 760 & $\mathbf{K r}$ \\
\hline Crysoberyi & $\mathrm{BeAl}_{2} \mathrm{O}_{4}$ & 750 & Kr; flashlamp; Hg \\
\hline Emerald & $\mathrm{Be}_{3} \mathrm{Al}_{2}\left(\mathrm{SiO}_{3}\right)_{6}$ & 750 & $2 \times \mathrm{Nd}: \mathrm{YAG}+\mathrm{SRS}$; flashlamp \\
\hline Scheelite & $\mathrm{ZnWO}_{4}$ & 990 & $\mathbf{K r}$ \\
\hline Fluoride perovskite & $\mathrm{KZnF}_{\mathbf{3}}$ & 790 & $\mathbf{K r}$ \\
\hline Fluoride gamet & $\mathrm{Li}_{3} \mathrm{Na}_{3} \mathrm{Ga}_{2} \mathrm{~F}_{12}$ & 765 & Pulsed-dye \\
\hline
\end{tabular}

02-50-0684-1983 


\section{Spectral properties of fluoride garnet}

${ }^{*} p(\mathrm{Cr})=2.1 \times 10^{20} \mathrm{ions} / \mathrm{cc}$

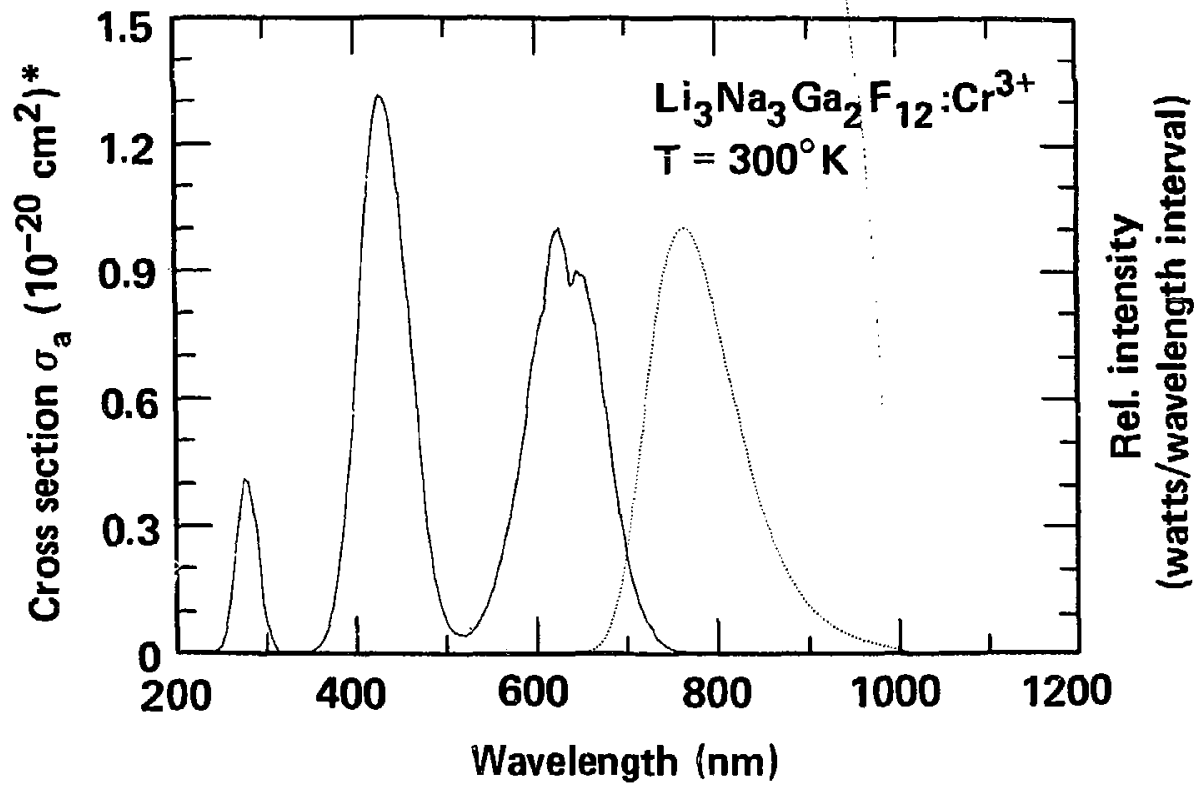



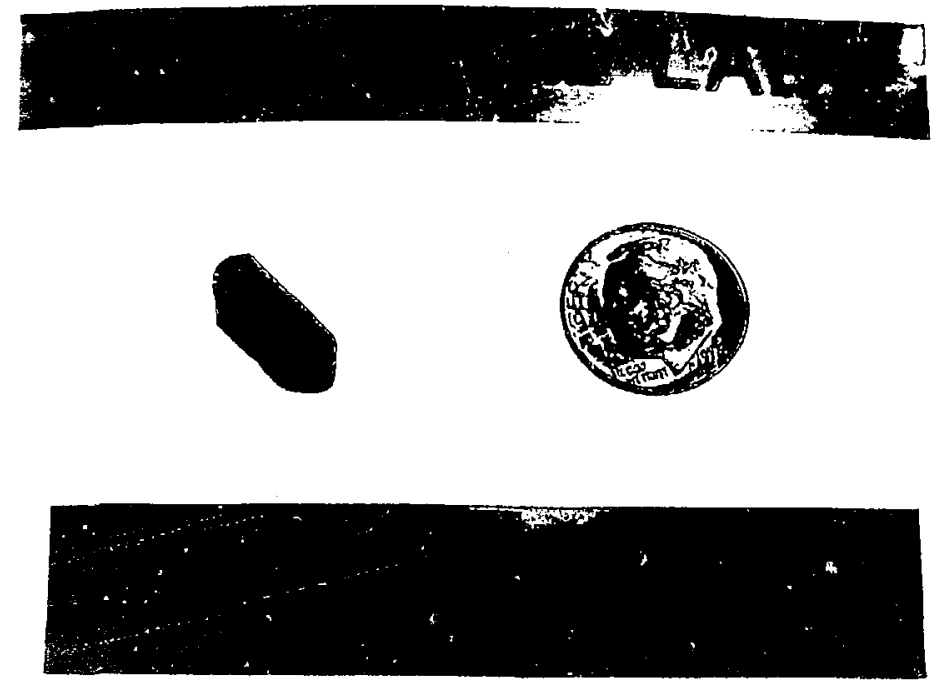

*Growth: H. Guggenheim (AT\&T - Bell, Labs) 


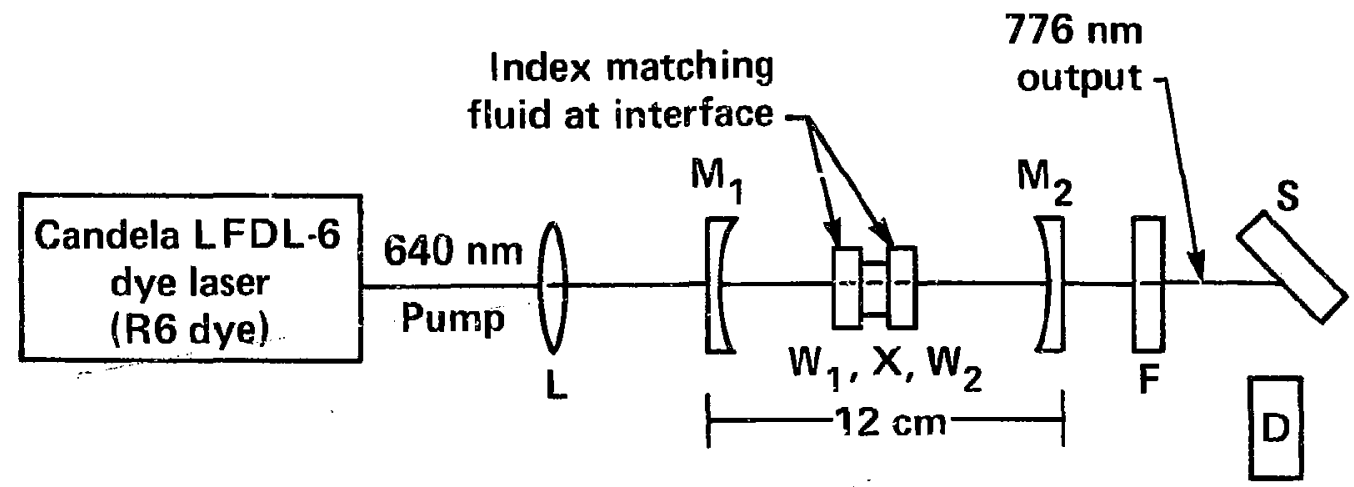

$X \quad \mathrm{Li}_{3} \mathrm{Na}_{3} \mathrm{Ga}_{2} \mathrm{~F}_{12}:$ Cr laser crystal $(0.5 \mathrm{~cm}$ thick)

$W_{1}, W_{2}$ Fused silica windows, AR coated outside

$M_{1}, M_{2} 25 \mathrm{~cm}$ radius mirrors, $\max R 750-800 \mathrm{~nm}$

L $\quad 25 \mathrm{~cm}$ focal length lens

F Long wavelength pass filter

S Diffuse scatterer

D Photodiode detector 


\section{Xenon lamp - fluoride perovskite coupling}

${ }^{*} \rho(\mathrm{Cr})=3.7 \times 10^{19} \mathrm{ions} / \mathrm{cc}$

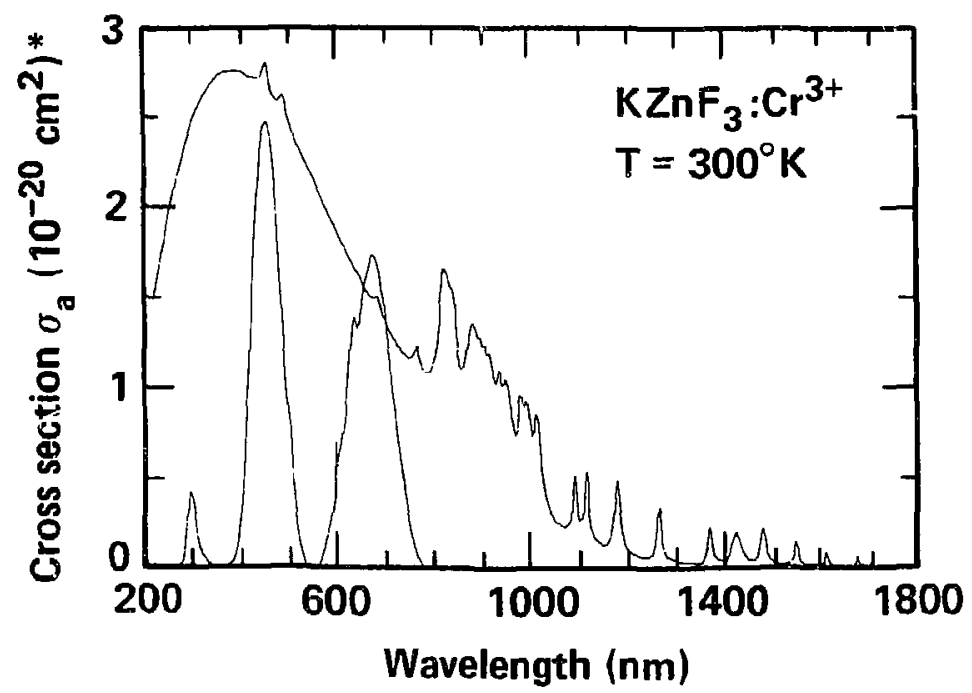

02-50-0684-1971 
Chromium doped fluoride perovskite laser*
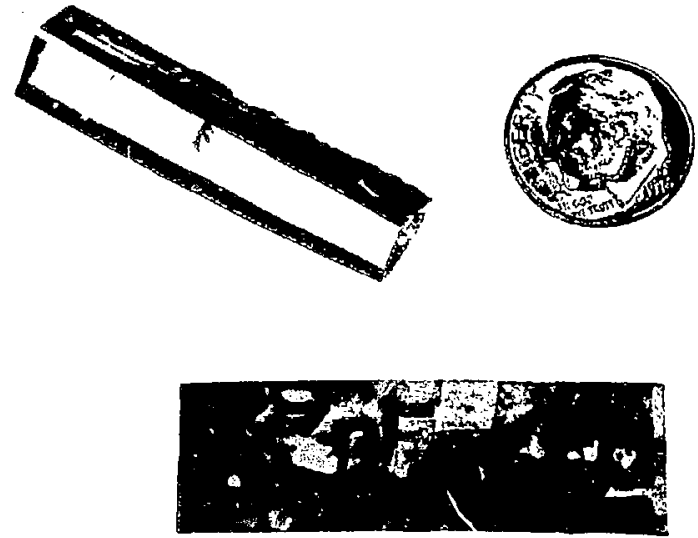

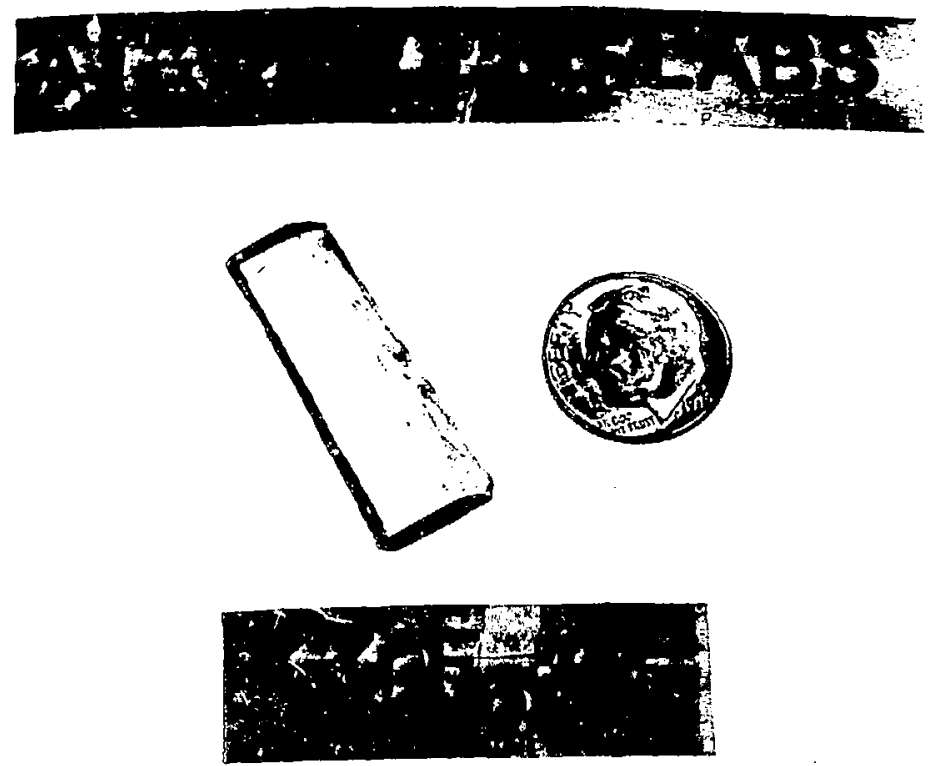

*Growth: H. Guggenheim (AT\&"- - Bell Labs) 
Set-up for fluoride perovskite laser experiment

$800 \mathrm{~nm}$

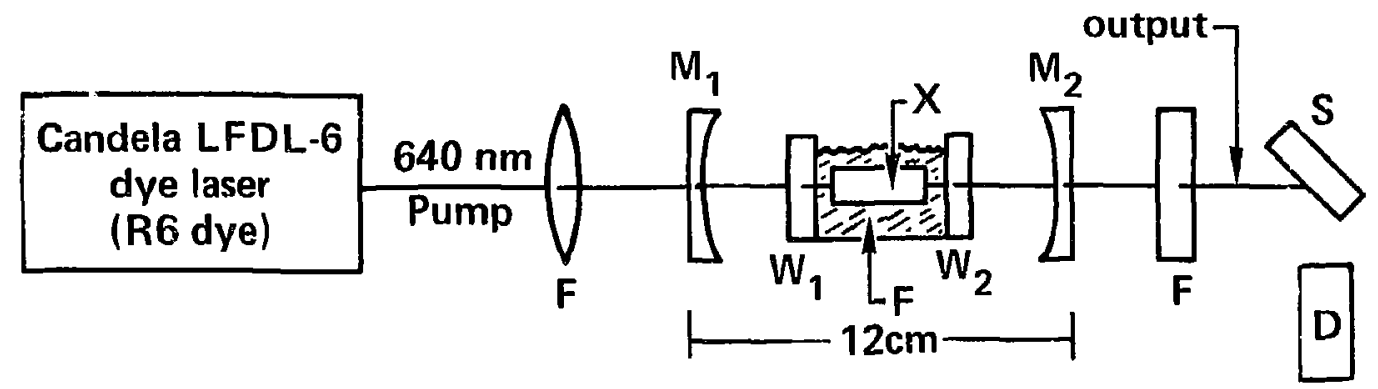

$\mathrm{X} \quad \mathrm{KZnF}_{3}:$ Cr laser crystal $(4 \mathrm{~cm}$ long)

$F \quad$ Index matching fluid

$W_{1}, W_{2}$ Fused silica windows, AR coated outside

$M_{1}, M_{2} 25 \mathrm{~cm}$ radius mirrors, $\max R 750-800 \mathrm{~nm}$

L $\quad 25 \mathrm{~cm}$ focal length lens

F Long wavelength pass filter

$S \quad$ Diffuse scatterer

D Photodiode detector 


\section{Laser parameters for $\mathrm{Cr}^{3+}-$ gain media}

\begin{tabular}{|l|l|c|c|c|c|c|c|}
\hline \multicolumn{1}{|c|}{ Crystal } & Name & $n$ & $\begin{array}{r}\tau_{f}(\mu \mathrm{s}) \\
300^{\circ} \mathrm{K}\end{array}$ & $\begin{array}{c}\widetilde{\Delta \nu} \\
\left(\mathrm{cm}^{-1}\right)\end{array}$ & $\begin{array}{c}\lambda_{\mathrm{p}} \\
(\mathrm{nm})\end{array}$ & $\begin{array}{c}\sigma_{\mathrm{p}}(\mathrm{pm}) \\
300^{\circ} \mathrm{K}\end{array}$ & $\begin{array}{l}\Gamma_{\text {sat }}^{\text {peak }} \\
\left(\mathrm{J} / \mathrm{cm}^{2}\right)\end{array}$ \\
\hline $\mathrm{BeAl}_{2} \mathrm{O}_{4}$ & Alexandrite & 1.74 & 240 & $\sim 1800$ & 750 & 0.6 & $\sim 44$ \\
$\mathrm{Ba}_{3} \mathrm{Al}_{2}\left(\mathrm{SiO}_{3}\right)_{6}$ & Emerald & 1.75 & 60 & $\sim 2000$ & 725 & 1.9 & $\sim 14$ \\
\hline $\mathrm{Gd}_{3} \mathrm{Ga}_{5} \mathrm{O}_{12}$ & GGG & 1.94 & 160 & $\sim 1600$ & 740 & 0.8 & $\sim 33$ \\
$\mathrm{Gd}_{3} \mathrm{Sc}_{2} \mathrm{Ga}_{3} \mathrm{O}_{12}$ & GSGG & 1.90 & 120 & $\sim 1700$ & 760 & 1.0 & $\sim 27$ \\
$\mathrm{Y}_{3} \mathrm{Ga}_{5} \mathrm{O}_{12}$ & YGG & 1.96 & 240 & $\sim 1300$ & 740 & 0.6 & $\sim 45$ \\
$\mathrm{Y}_{3} \mathrm{Sc}_{2} \mathrm{Ga}_{3} \mathrm{O}_{12}$ & YSGG & 1.86 & $1140)$ & $\sim 1700$ & 760 & 0.9 & $\sim 28$ \\
$\mathrm{La}_{3} \mathrm{Lu}_{2} \mathrm{Ga}_{3} \mathrm{O}_{12}$ & LLGG & $11.95)$ & 70 & $\sim 1750$ & 820 & 1.9 & $\sim 13$ \\
\hline $\mathrm{K}_{2} \mathrm{NaScF}_{6}$ & Elpasolite & 1.44 & 280 & -2000 & 775 & 0.7 & $\sim 37$ \\
$\mathrm{~K}_{2} \mathrm{LiGaF}_{6}$ & & 1.43 & 330 & $\sim 1600$ & 760 & 0.7 & $\sim 37$ \\
\hline $\mathrm{Li}_{3} \mathrm{Na}_{3} \mathrm{Ga}_{2} \mathrm{~F}_{12}$ & Gamet & 1660 & 755 & 0.5 & $\sim 57$ \\
\hline $\mathrm{KZnF}_{3}$ & Perovskite & 1.46 & 176 & $\sim 1700$ & 795 & 1.3 & $\sim 19$ \\
\hline
\end{tabular}

"Brauch and Dürr, Opt. Comm. 49, 61 (1984); inconsistent with $\sigma_{p}=\lambda_{p}^{2} / 8 \pi \mathrm{cn}^{2} \widetilde{\Delta \nu \tau_{f}}$ 


\section{Parameters of $\mathrm{Cr}^{3+}\left(3 d^{3}\right)$ laser gain media}

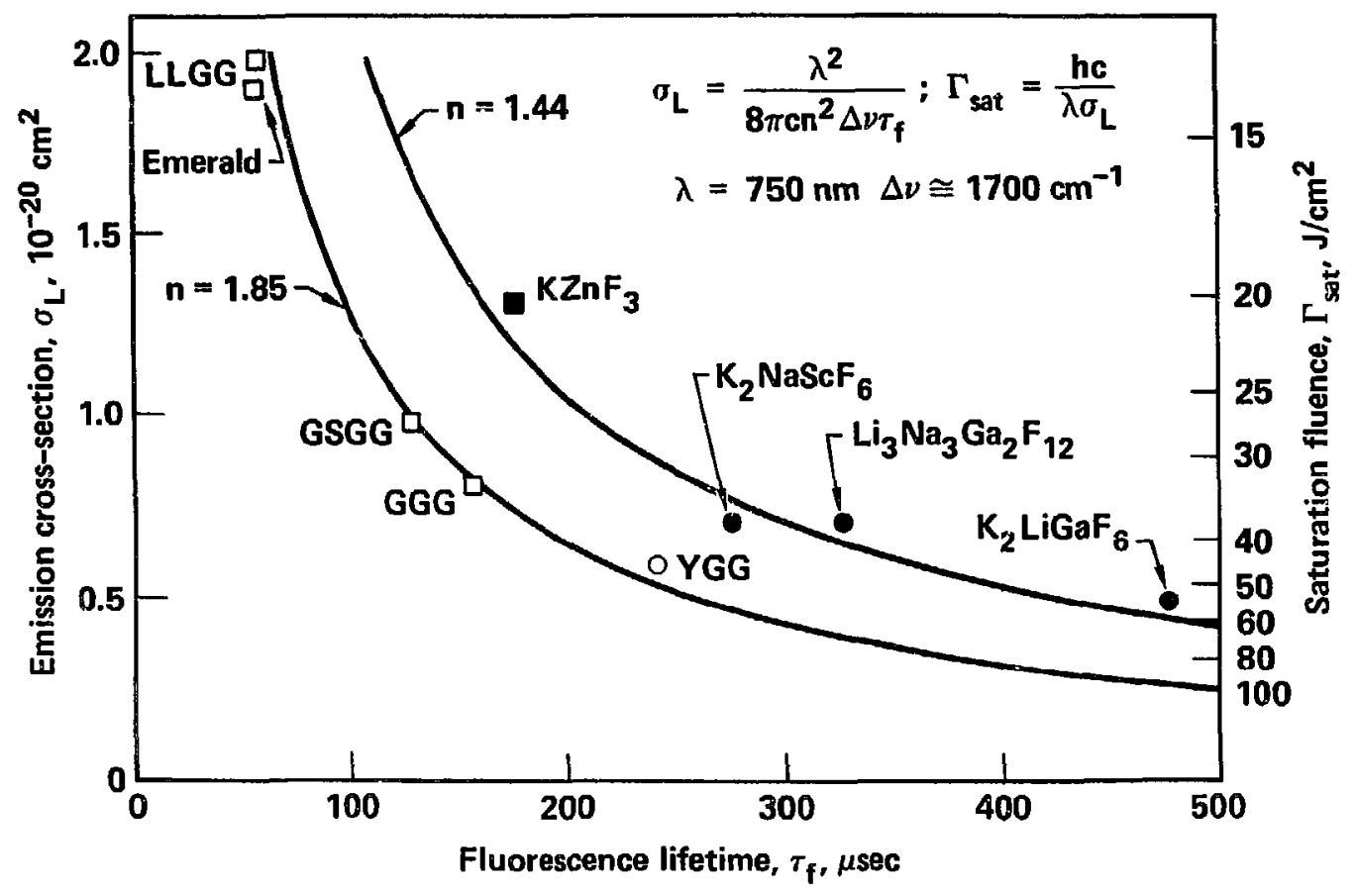




\section{Developments expected in '85}

Co-doped (Cr:Nd) materials

- Scaled-growth of GSGG - $10 \times 20 \mathrm{~cm}^{2}$ slabs

- Increased efficiency $>6 \%$

- Evaluation of alternative oxide garnets

- Gd(GaCaZr) GaG

- GdInGaG

- GdScAIG

Chromium-doped tunable materials

- Materials with larger stimulated emission cross-sections $\left(\sigma \geqslant 2 \times 10^{-20} ; \Gamma_{\text {sat }}<15 \mathrm{~J} / \mathrm{cm}^{2}\right)$ 